Research Article

\title{
Experiment and Analysis of Active Vibration Suppression via an Absorber with a Tunable Delay
}

\author{
Yixia Sun \\ School of Mechanical and Automotive Engineering, Shanghai University of Engineering Science, Shanghai 201620, China \\ Correspondence should be addressed to Yixia Sun; sunyixia@sues.edu.cn
}

Received 4 February 2020; Revised 20 August 2020; Accepted 25 August 2020; Published 3 September 2020

Academic Editor: Guo-Qing Zhang

Copyright (C) 2020 Yixia Sun. This is an open access article distributed under the Creative Commons Attribution License, which permits unrestricted use, distribution, and reproduction in any medium, provided the original work is properly cited.

\begin{abstract}
A time-delayed absorber is utilized to suppress the vibration of a primary system excited by a simple harmonic force. The inherent and intentional time delays in the feedback control loop are taken into consideration. The value of the former is fixed, while the value of the latter is tunable in the controller. To begin with, the mechanical model of the system is established and the acceleration transfer functions of the system are derived. Consequently, the stability analysis of the coupled system is carried out. Finally, the experimental studies on the performance of the time-delayed absorber are conducted. Both experimental and theoretical results show that the time-delayed absorber with proper values of feedback gain coefficient and intentional time delay greatly suppresses the vibration of the primary system. The numerical results validate the correctness of the experimental and theoretical ones.
\end{abstract}

\section{Introduction}

Time delay is inherent in the active control loop, which is derived from the signal acquisition and processing, filtering, the action of the actuator, etc [1]. In fact, time delay exists in various research fields, such as aerospace engineering [2], medicine [3], communication [4], and machining [5].

Originally, the time delay was taken as a negative factor in the active control. It may result in the degradation of the control performance and the instability of the controlled system. Therefore, several methods were employed to compensate the adverse influence of time delay, such as phase-shift method [6], Smith predictor method [7], and Pade approximation method [8]. However, in the past three decades, lots of works have showed that intentional introduction of time delay in the feedback control loop benefits control effect. The time-delayed feedback control technique has been widely used in controlling chaos [9], improving system stability [10], and vibration control performance $[11,12]$.

A time-delayed absorber is a new technique in the field of active vibration control. The key idea of the time-delayed absorber is the introduction of an actuator controlled via timedelayed feedback control. In 1994, Olgac and Holm-Hansen
[13] firstly presented the concept of Delayed Resonator (DR). When the proportionality gain and time delay are properly selected, the resonator moves all vibration from a primary system at its point of attachment. After that, Olgac et al. conducted in-depth studies on the application of DR [14-16]. Zhao et al. [17] investigated the effect of a nonlinear timedelayed absorber on suppressing the vertical vibration of a primary system when the primary resonance and $1: 1$ internal resonance occurred simultaneously. Mohanty and Dwivedy [18] studied the vibration control performance of a piezoelectric-based nonlinear vibration absorber using time-delayed acceleration feedback when the nonlinear primary system is harmonically excited. Sun and Song [19] discussed the timedelayed active control of vibration absorbers attached to a continuous beam structure. It is worth noting that extensive studies on the time-delayed absorber focus on theoretical analysis and numerical simulation. Few efforts have been devoted to experiments $[20,21]$.

In our previous work [22], it is demonstrated that the time-delayed absorber with proper feedback gain coefficient and inherent time delay greatly reduces the vibration amplitude of a primary system. Motivated by this finding, an intentional time delay is introduced in the controller and the vibration suppression effect of the time-delayed absorber 
with the inherent and the intentional time delays is studied in this paper.

The present paper is organized as follows. The mechanical model and stability analysis are shown in Section 2. In Sections 3, the experimental studies are conducted. The effects of the feedback gain coefficient and intentional time delay on vibration suppression effect of the time-delayed absorber are, respectively, investigated. Conclusions are presented in Section 4.

\section{Modeling and Stability Analysis}

2.1. Modeling. Figure 1 illustrates the mechanical model of the 2-dof coupled system [23]. The coupled system consists of a time-delayed absorber and a primary system. $m_{1}$ and $m_{2}$ represent the mass of the absorber and the primary system, respectively. The motions of the absorber and the primary system are denoted by $x_{1}$ and $x_{2}$, respectively. $k_{1}$ and $k_{2}$ represent the linear stiffness coefficients. $c_{1}$ and $c_{2}$ represent the viscous damping coefficients. An actuator mounted between the primary mass and the absorber mass provides the time-delayed feedback control force.

Assuming that simply harmonic excitation is applied to the primary mass, the governing equations of the coupled system are given by

$$
\begin{aligned}
& m_{1} \ddot{x}_{1}+k_{1}\left(x_{1}-x_{2}\right)+c_{1}\left(\dot{x}_{1}-\dot{x}_{2}\right)-k_{c} x_{c}=0, \\
& m_{2} \ddot{x}_{2}+k_{2} x_{2}+c_{2} \dot{x}_{2}+k_{1}\left(x_{2}-x_{1}\right)+c_{1}\left(\dot{x}_{2}-\dot{x}_{1}\right)+k_{c} x_{c} \\
& =f \sin (\omega t),
\end{aligned}
$$

where $k_{c} x_{c}$ is the time-delayed feedback control force, $k_{c}$ is the stiffness coefficient of the actuator, and $x_{c}$ is the control signal with the following form:

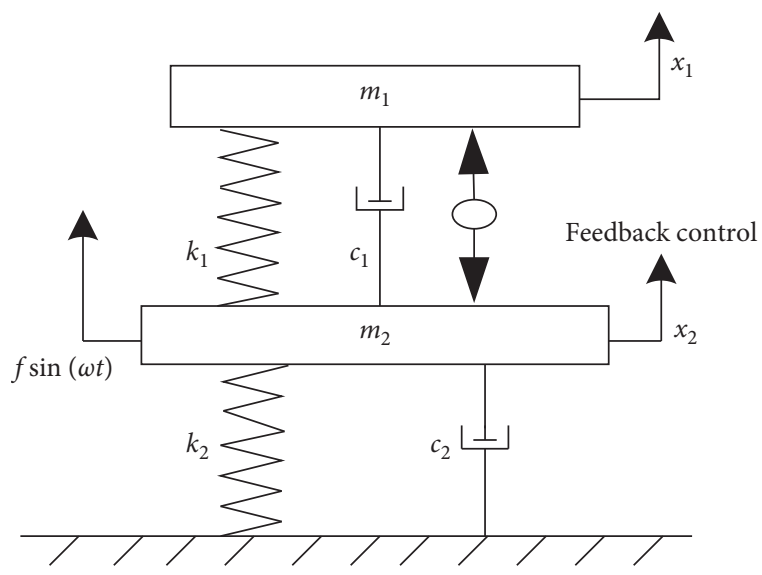

Figure 1: Mechanical model of the coupled system.

$$
\begin{aligned}
x_{c}= & \alpha_{1}\left[x_{1}\left(t-\tau_{1}\right)-x_{2}\left(t-\tau_{1}\right)\right] \\
& +\alpha_{2}\left[x_{1}\left(t-\tau_{1}-\tau_{2}\right)-x_{1}\left(t-\tau_{1}\right)\right],
\end{aligned}
$$

where $\tau_{1}$ and $\tau_{2}$, respectively, represent the inherent and the intentional time delays in the feedback control loop. The value of $\tau_{1}$ is constant, while the value of $\tau_{2}$ is tunable in the controller. Instead of eliminating, compensating for, and even ignoring the presence of $\tau_{1}$, it is thought that $\tau_{2}$ together with $\tau_{1}$ leads to the final control effect. $\alpha_{1}$ and $\alpha_{2}$ represent amplification factors of the control signal. The time-delayed feedback disappears and the time-delayed absorber degrades into the passive one when $\alpha_{1}=\alpha_{2}=0$.

Substituting equation ( 3 ) into equations (1) and (2) gives

$$
\begin{aligned}
& m_{1} \ddot{x}_{1}+k_{1}\left(x_{1}-x_{2}\right)+c_{1}\left(\dot{x}_{1}-\dot{x}_{2}\right)-g_{1}\left[x_{1}\left(t-\tau_{1}\right)-x_{2}\left(t-\tau_{1}\right)\right]-g_{2}\left[x_{1}\left(t-\tau_{1}-\tau_{2}\right)-x_{1}\left(t-\tau_{1}\right)\right]=0, \\
& m_{2} \ddot{x}_{2}+k_{2} x_{2}+c_{2} \dot{x}_{2}+k_{1}\left(x_{2}-x_{1}\right)+c_{1}\left(\dot{x}_{2}-\dot{x}_{1}\right)+g_{1}\left[x_{1}\left(t-\tau_{1}\right)-x_{2}\left(t-\tau_{1}\right)\right]+g_{2}\left[x_{1}\left(t-\tau_{1}-\tau_{2}\right)-x_{1}\left(t-\tau_{1}\right)\right] \\
& =f \sin (\omega t),
\end{aligned}
$$

where $g_{1}=\alpha_{1} k_{c}$ and $g_{2}=\alpha_{2} k_{c}$ represent feedback gain coefficients.

The external excitation can be rewritten as

$$
f \sin (\omega t)=f e^{j \omega t} .
$$

The solutions of equations (4) and (5) are considered to

$$
\left[\begin{array}{l}
x_{1} \\
x_{2}
\end{array}\right]=\left[\begin{array}{l}
\bar{x}_{1} \\
\bar{x}_{2}
\end{array}\right] e^{j \omega t}
$$

where $\bar{x}_{1}$ and $\bar{x}_{2}$ are complex.

Substituting equations (6) and (7) into equations (4) and (5), one gets be

$$
\left[\begin{array}{l}
\bar{x}_{1} \\
\bar{x}_{2}
\end{array}\right]=\frac{f}{\Delta(\omega)}\left[\begin{array}{c}
k_{1}+j c_{1} \omega-g_{1} e^{-j \omega \tau_{1}} \\
-m_{1} \omega^{2}+k_{1}+j c_{1} \omega+\left(g_{2}-g_{1}\right) e^{-j \omega \tau_{1}}-g_{2} e^{-j \omega\left(\tau_{1}+\tau_{2}\right)}
\end{array}\right]
$$


where

$$
\begin{aligned}
\Delta(\omega)= & {\left[-m_{1} \omega^{2}+k_{1}+j c_{1} \omega+\left(g_{2}-g_{1}\right) e^{-j \omega \tau_{1}}-g_{2} e^{-j \omega\left(\tau_{1}+\tau_{2}\right)}\right] \times\left[-m_{2} \omega^{2}+k_{2}+k_{1}+j\left(c_{1}+c_{2}\right) \omega-g_{1} e^{-j \omega \tau_{1}}\right] } \\
& -\left[k_{1}+j c_{1} \omega-g_{1} e^{-j \omega \tau_{1}}\right]\left[k_{1}+j c_{1} \omega-\left(g_{1}-g_{2}\right) e^{-j \omega \tau_{1}}-g_{2} e^{-j \omega\left(\tau_{1}+\tau_{2}\right)}\right] .
\end{aligned}
$$

To facilitate the following analysis, new variables $H_{1}$ and $\mathrm{H}_{2}$ are defined as follows:

$$
H_{i}=\frac{\left\|\ddot{x}_{i}\right\|}{f}, \quad(i=1,2),
$$

where $H_{1}$ and $H_{2}$ represent the acceleration transfer functions of the absorber and the primary system, respectively.

It is seen from equation (10) that $H_{1}$ and $H_{2}$ describe the vibration intensity of the absorber and the primary system, respectively. Hence, for fixed values of physical parameters and inherent time delay, it is available to evaluate the vibration suppression effect of the time-delayed absorber by comparing the value of $H_{2}$ under different values of $g_{1}, g_{2}$, and $\tau_{2}$.

2.2. Stability Analysis. It is known that the values of feedback gain coefficients and time delays determine the stability of the system when the physical parameters of the coupled system are fixed. Hence, it is necessary to analyze the stability of the system before the experiments are carried out.

In Laplace domain, equations (4) and (5) become

$$
\mathbf{A}(s) \mathbf{X}(s)=\mathbf{F}(s),
$$

where

$$
\begin{aligned}
& \mathbf{A}(s)=\left[\begin{array}{cc}
m_{1} s^{2}+k_{1}+c_{1} s+\left(g_{2}-g_{1}\right) e^{-\tau_{1} s}-g_{2} e^{-\left(\tau_{1}+\tau_{2}\right) s} & -k_{1}-c_{1} s+g_{1} e^{-\tau_{1} s} \\
-k_{1}-c_{1} s-\left(g_{2}-g_{1}\right) e^{-\tau_{1} s}+g_{2} e^{-\left(\tau_{1}+\tau_{2}\right) s} & m_{2} s^{2}+k_{1}+k_{2}+\left(c_{1}+c_{2}\right) s-g_{1} e^{-\tau_{1} s}
\end{array}\right], \\
& \mathbf{X}(s)=\left[X_{1}(s), X_{2}(s)\right]^{T}, \\
& \mathbf{F}(s)=[0, F(s)]^{T} .
\end{aligned}
$$

The characteristic equation of the coupled system is det (A) $=0$; that is,

$$
P_{0}(s)+P_{1}(s) e^{-\tau_{1} s}+P_{2}(s) e^{-\left(\tau_{1}+\tau_{2}\right) s}=0,
$$

where

$$
\begin{aligned}
& P_{0}(s)=k_{1} k_{2}+\left(c_{2} k_{1}+c_{1} k_{2}\right) s+\left(c_{1} c_{2}+k_{1} m_{1}+k_{2} m_{1}+k_{1} m_{2}\right) s^{2}+\left(c_{1} m_{1}+c_{2} m_{1}+c_{1} m_{2}\right) s^{3}+m_{1} m_{2} s^{4}, \\
& P_{1}(s)=\left(g_{2}-g_{1}\right) k_{2}+\left(-g_{1} m_{1}-g_{1} m_{2}+g_{2} m_{2}\right) s^{2}+c_{2}\left(g_{2}-g_{1}\right) s, \\
& P_{2}(s)=-g_{2} k_{2}-c_{2} g_{2} s-g_{2} m_{2} s^{2} .
\end{aligned}
$$

The coupled system is stable if and only if all characteristic roots of equation (13) have negative real parts. When equation (13) has a pure imaginary root, stability switch may occur. Let

$$
s=\omega_{c} i, \quad\left(\omega_{c}>0\right) .
$$

Substituting equation (15) into equation (13) and separating the real and the imaginary parts, one obtains

$$
\begin{aligned}
& {\left[\left(g_{2}-g_{1}\right) k_{2}+\left(-g_{2} m_{2}+g_{1} m_{1}+g_{1} m_{2}\right) \omega_{c}^{2}\right] \cos \left(\omega_{c} \tau_{1}\right)-c_{2} \omega_{c}\left(g_{1}-g_{2}\right) \sin \left(\omega_{c} \tau_{1}\right)-c_{2} \omega_{c} g_{2} \sin \left[\omega_{c}\left(\tau_{1}+\tau_{2}\right)\right]} \\
& \quad+g_{2}\left(m_{2} \omega_{c}^{2}-k_{2}\right) \cos \left[\omega_{c}\left(\tau_{1}+\tau_{2}\right)\right]+k_{1} k_{2}+m_{1} m_{2} \omega_{c}^{4}-\left(c_{1} c_{2}+k_{1} m_{1}+k_{2} m_{1}+k_{1} m_{2}\right) \omega_{c}^{2}=0, \\
& {\left[g_{2}\left(m_{2} \omega_{c}^{2}-k_{2}\right)+g_{1}\left(k_{2}-m_{1} \omega_{c}^{2}-m_{2} \omega_{c}^{2}\right)\right] \sin \left(\omega_{c} \tau_{1}\right)+c_{2}\left(g_{2}-g_{1}\right) \omega_{c} \cos \left(\omega_{c} \tau_{1}\right)-c_{2} g_{2} \omega_{c} \cos \left[\omega_{c}\left(\tau_{1}+\tau_{2}\right)\right]} \\
& \quad+g_{2}\left(k_{2}-m_{2} \omega_{c}^{2}\right) \sin \left[\omega_{c}\left(\tau_{1}+\tau_{2}\right)\right]+\left(c_{2} k_{1}+c_{1} k_{2}\right) \omega_{c}-\left(c_{2} m_{1}+c_{1} m_{1}+c_{1} m_{2}\right) \omega_{c}^{3}=0 .
\end{aligned}
$$



has

Using $\sin ^{2}\left[\omega_{c}\left(\tau_{1}+\tau_{2}\right)\right]+\cos ^{2}\left[\omega_{c}\left(\tau_{1}+\tau_{2}\right)\right]=1$, one

$$
d_{8} \omega_{c}^{8}+d_{6} \omega_{c}^{6}+d_{5} \omega_{c}^{5}+d_{4} \omega_{c}^{4}+d_{3} \omega_{c}^{3}+d_{2} \omega_{c}^{2}+d_{1} \omega_{c}+d_{0}=0
$$

where the expressions of $d_{l}(l=1,2,3,4,5,6,8)$ are given in Appendix.

Since equation (18) is a high-order equation with transcendental terms, the values of $\omega_{c}$ are obtained by numerical calculation. For fixed values of $\tau_{1}$ and certain values of feedback gain coefficients $g_{1}=g_{10}$ and $g_{2}=g_{20}, N$ is assigned to denote the number of the positive real roots of equation (18). When $N=0$, there is no stability switch. In other words, the stability of the system remains the same for all $\tau_{2} \in \mathfrak{R}^{+}$. When $N \neq 0$, we have $\omega_{c 1}, \omega_{c 2}, \ldots, \omega_{c n}$. For each $\omega_{c j}(j=1,2, \ldots, N)$, infinite $\tau_{2}$ values $\tau_{21}, \tau_{22}, \ldots, \tau_{2 \infty}$ are determined by solving equations (16) and (17). The transition direction of the roots at $\omega_{c j}$ as $\tau_{2}$ increases from $\tau_{2 p}-\varepsilon$ to $\tau_{2 p}+\varepsilon(0<\varepsilon \ll 1, p=1,2, \ldots, \infty)$ is decided by the expression:

$$
R D=\operatorname{sgn}\left[\operatorname{Re}\left(\frac{\partial s}{\partial \tau_{2}} \mid s=\omega_{c j} i\right)\right],
$$

and +1 and -1 values of $\mathrm{RD}$ represent destabilizing and stabilizing transitions, respectively.

Through the above computation, the stable and unstable ranges of $\tau_{2}$ for $g_{1}=g_{10}$ and $g_{2}=g_{20}$ are divided. Performing the same procedure for other values of $g_{1}$ and $g_{2}$, the stable and unstable regions in the space $\left(g_{1}, g_{2}, \tau_{2}\right)$ are plotted.

\section{Experimental Studies on the Time- Delayed Absorber}

3.1. Experimental Setup. The photo of the experimental setup is shown in Figure 2. The absorber mass (1) is attached to the primary mass (2) by five sheets of steel. A servo motor (3) is fixed on the primary mass. The primary mass is connected to the base (5) by four sheets of steel. The controlled steel sheet (4) acts as the actuator and exerts the control force, whose lower end is linked to the shaft of the servo motor by a wire rope. A shaker (6) provides a horizontal exciting force to the primary mass.

Figure 3 illustrates the schematic of time-delayed feedback control. A shaker (5) provides a sinusoidal excitation force to the primary mass (2). The amplitude and frequency of the excitation force can be set in $M+p$ vibpilot. A force sensor (6) and two acceleration sensors (7) and (8) are used to monitor the excitation force and the responses of the absorber and the primary mass, respectively. The time-delayed feedback control loop is described as follows:

Step 1: the acceleration signals of the absorber mass (1) and the primary mass enter into signal conditioning instrument, in which the functions of signal amplification and low-pass filtering are achieved to improve the signal-to-noise ratio.
Step 2: the processed signals go into the voltage lifting device, where the voltage of the input signal is raised 5 volts.

Step 3: the raised voltage signal enters into Trio motion controller, where control commands are written. The values of feedback control feedback gains and intentional time delay can be adjusted in the control commands.

Step 4: the control commands are transferred into the servo controller, which guides the shaft rotation of the servo motor (3).

Step 5: driven by the rotation of the servo motor shaft, the lower end of the controlled steel sheet (4) realizes the horizontal reciprocating motion and applies the time-delayed feedback control force.

As a preliminary, the values of the physical parameters of the 2-dof coupled system need to be identified when the time-delayed feedback control is absent (i.e., $\alpha_{1}=\alpha_{2}=0$ in equations (4) and (5)). Appling a sine swept excitation to the primary mass, the acceleration transfer function curves of the coupled system are obtained. Using the least-squares method, the values of the physical parameters are identified, as shown in Table 1 . In addition, the value of inherent time delay $\tau_{1}$ is identified to be $63 \mathrm{~ms}$ [22].

Figure 4 shows the comparison of the experimental and the theoretical results of the acceleration transfer functions, where $\Omega=(\omega / 2 \pi)$ denotes the excitation frequency. It is seen that the passive absorber is most effective when $\Omega=9.63 \mathrm{~Hz}$. When the excitation frequency is disturbed and deviates from $9.63 \mathrm{~Hz}$, vibration control performance of the passive absorber deteriorates. In this case, the timedelayed feedback control is introduced to transform the passive absorber into a time-delayed one. Proper values of intentional time delay and feedback gain coefficients are adapted to improve the vibration suppression effect of the absorber.

3.2. Experimental Results. In this subsection, the effects of $\alpha_{2}$ and $\tau_{2}$ on the vibration suppression effect of the timedelayed absorber are, respectively, discussed when $\Omega=9.75 \mathrm{~Hz}$ and $10 \mathrm{~Hz}$.

Figure 5 shows the stability charts of the coupled system for $\tau_{2}=50 \mathrm{~ms}, \tau_{2}=30 \mathrm{~ms}$, and $\alpha_{2}=0.5$, respectively. In Figures 5(a) and (b), the coupled system is stable in region II, while it is unstable in regions I and III. In Figure 5(c), the coupled system is stable in region I, while it is unstable in regions II-VI.

\subsubsection{Effect of $\alpha_{2}$}

Case 1. $\Omega=9.75 \mathrm{~Hz}, \alpha_{1}=-0.4$, and $\tau_{2}=50 \mathrm{~ms}$.

Figure 6 shows how the acceleration transfer functions of the system $H_{i}(i=1,2)$ change as a function of $\alpha_{2}$ for $\Omega=9.75 \mathrm{~Hz}, \alpha_{1}=-0.4$, and $\tau_{2}=50 \mathrm{~ms}$. Obviously, the experimental results agree well with the theoretical ones. Figure 6(a) indicates that the value of $H_{1}$ monotonically 


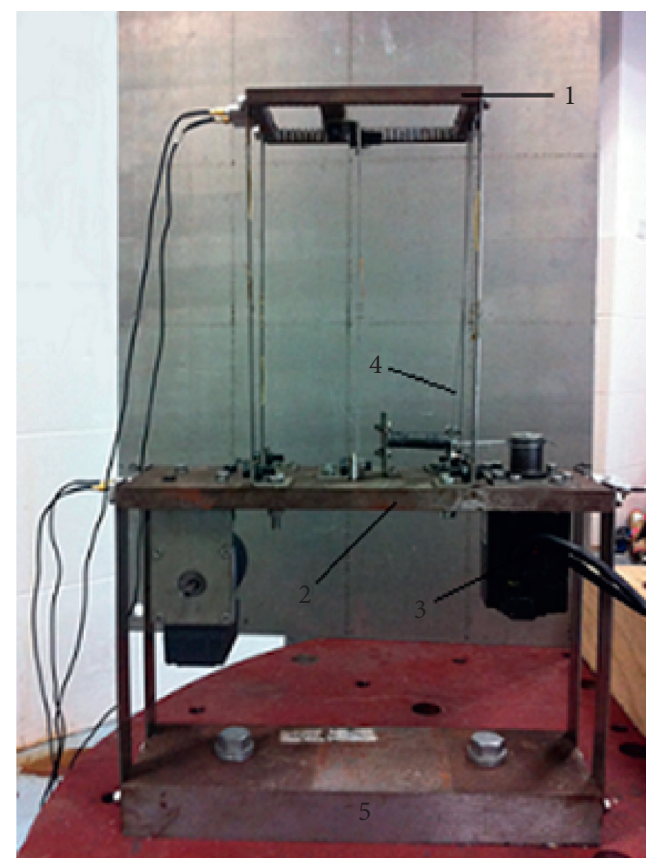

(a)

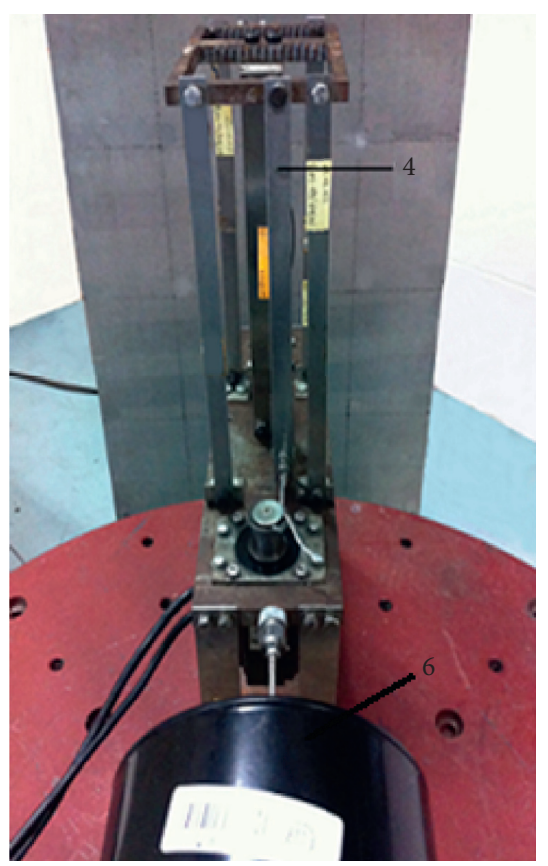

(b)

Figure 2: Photo of experiment setup: (a) front view; (b) right view (1: absorber mass, 2: primary mass, 3: servo motor, 4: controlled steel sheet, 5: base, and 6: shaker).

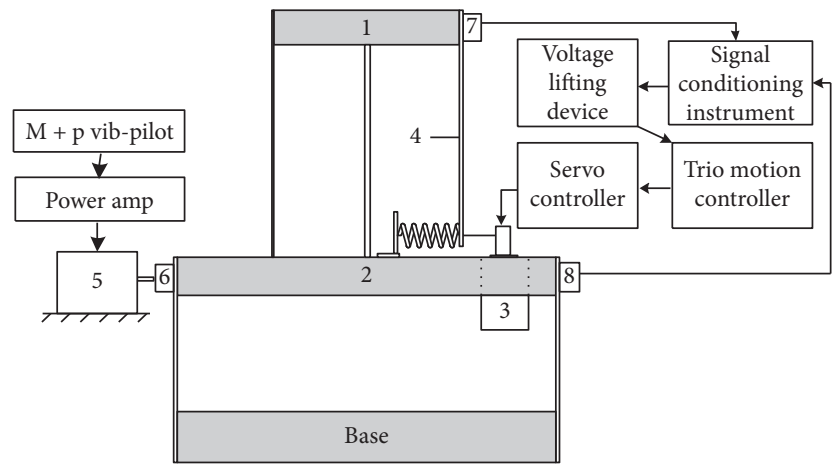

Figure 3: Schematic of time-delayed feedback control (1: absorber mass, 2: primary mass, 3: servo motor, 4: controlled steel sheet, 5: shaker, 6: force sensor, and 7 and 8: acceleration sensors).

TABle 1: Physical parameters of the coupled system.

\begin{tabular}{lcccccc}
\hline$m_{1}(\mathrm{~kg})$ & $m_{2}(\mathrm{~kg})$ & $k_{1}(\mathrm{~N} / \mathrm{m})$ & $k_{2}(\mathrm{~N} / \mathrm{m})$ & $k_{c}(\mathrm{~N} / \mathrm{m})$ & $c_{1}(\mathrm{Ns} / \mathrm{m})$ & $c_{2}(\mathrm{Ns} / \mathrm{m})$ \\
\hline 0.667 & 6.8 & 2431.72 & 13502.4 & 198.96 & 0.17 & 1 \\
\hline
\end{tabular}

increases with the increase of $\alpha_{2}$. As shown in Figure 6(b), the value of the $\mathrm{H}_{2}$ decreases firstly and then increases as $\alpha_{2}$ increases. The minimum of $\mathrm{H}_{2}$ occurs for $\alpha_{2}=-0.33$.

Figure 7 shows the measured time histories of excitation force and system accelerations. The total settling time is $144 \mathrm{~s}$. The time-delayed feedback control is activated at $t=29 \mathrm{~s}$. After a short transient, the amplitude of the excitation force and system acceleration responses reaches fixed values. It is found that although the amplitude of the excitation force increases, the acceleration amplitude of the primary system decreases sharply. The time-delayed feedback control is deactivated at $t=113 \mathrm{~s}$ and the coupled system returns to the initial uncontrolled state.

Table 2 shows the effect of $\alpha_{2}$ on the vibration suppression effect for $\Omega=9.75 \mathrm{~Hz}, \alpha_{1}=-0.4$, and $\tau_{2}=50 \mathrm{~ms}$. When the passive absorber works, the value of $\mathrm{H}_{2}$ is 


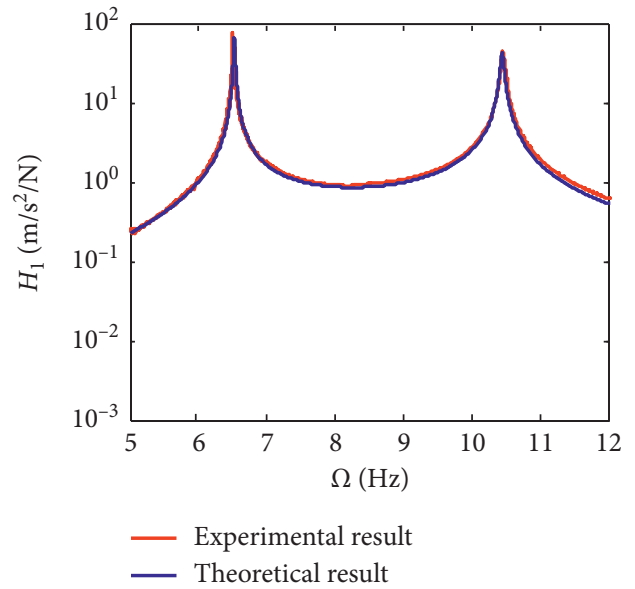

(a)

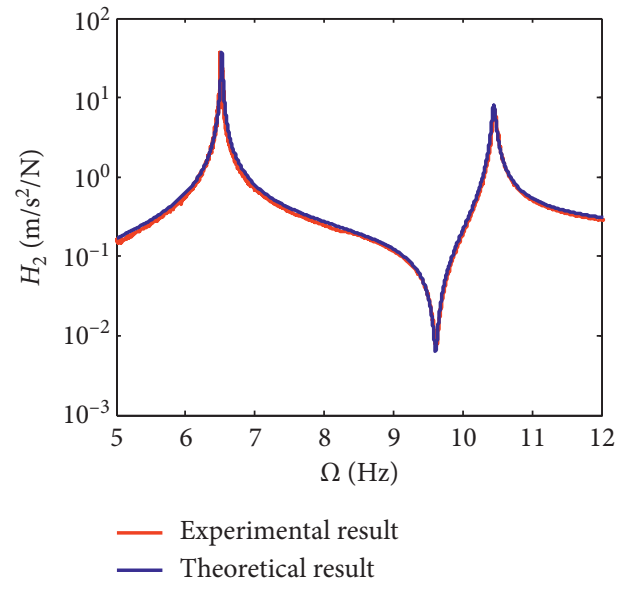

(b)

Figure 4: Comparison of the experimental and theoretical results of $H_{i}(i=1,2)$ : (a) absorber and (b) primary system.

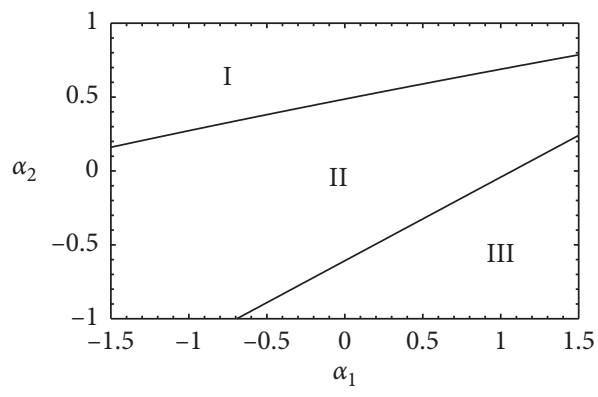

(a)

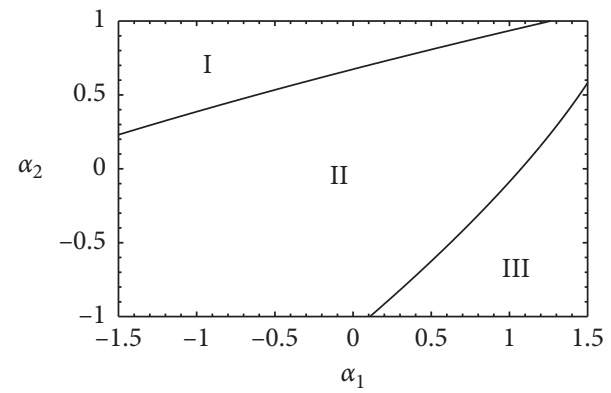

(b)

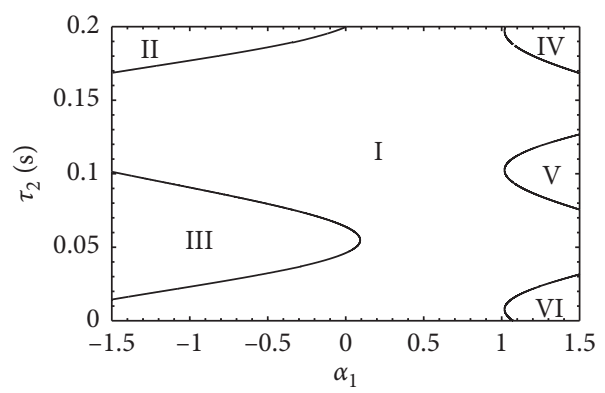

(c)

FIgURE 5: Stability charts of the coupled system. (a) $\tau_{2}=50 \mathrm{~ms}$. (b) $\tau_{2}=30 \mathrm{~ms}$. (c) $\alpha_{2}=0.5$.

$0.037 \mathrm{~m} / \mathrm{s}^{2} / \mathrm{N}$. When the time-delayed feedback control is activated, the value of $H_{2}$ decreases $45.95 \%$ for $\alpha_{1}=-0.4$ and $\alpha_{2}=-0.33$ and increases $159.46 \%$ for $\alpha_{1}=-0.4$ and $\alpha_{2}=-0.055$. It is drawn from Table 2 and Figure 6 that the vibration suppression effect of the time-delayed absorber is superior to that of the passive one for $\alpha_{2} \in[-0.495,-0.275]$.

Hereinafter,

$D=\left(\left(\left.H_{2}\right|_{\alpha_{1} \neq 0, \alpha_{2} \neq 0}-\left.H_{2}\right|_{\alpha_{1}=\alpha_{2}=0}\right) /\left.H_{2}\right|_{\alpha_{1}=\alpha_{2}=0}\right) \times 100 \%$.

To verify the experimental and theoretical results, numerical results are obtained by numerical integration of equations (4) and (5). Figure 8 shows the numerical simulations of system responses for $\Omega=9.75 \mathrm{~Hz}$ and $f=1 \mathrm{~N}$. From the comparison between Figures 6 and 8, the numerical results agree well with experimental and theoretical ones.

Case 2. $\Omega=10 \mathrm{~Hz}, \alpha_{1}=0.25$, and $\tau_{2}=30 \mathrm{~ms}$.

The variations of $H_{i}(i=1,2)$ versus $\alpha_{2}$ for $\Omega=10 \mathrm{~Hz}$, $\alpha_{1}=0.25$, and $\tau_{2}=30 \mathrm{~ms}$ are shown in Figure 9. It can be concluded from the experimental results that the value of $\mathrm{H}_{2}$ is maximal for $\alpha_{2}=-0.065$ and minimal for $\alpha_{2}=-0.455$.

Figure 10 shows the measured time histories of excitation force and system accelerations before and after the timedelayed feedback control. It is seen that after the feedback control is exerted, the amplitude of the excitation force increases $46.3 \%$, whereas the acceleration amplitudes of the 


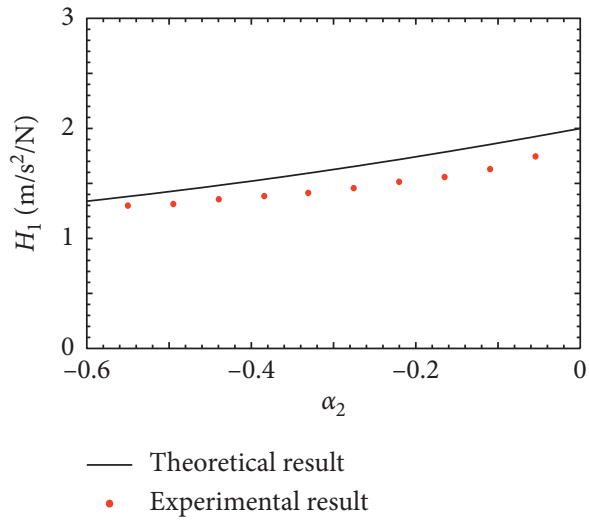

(a)

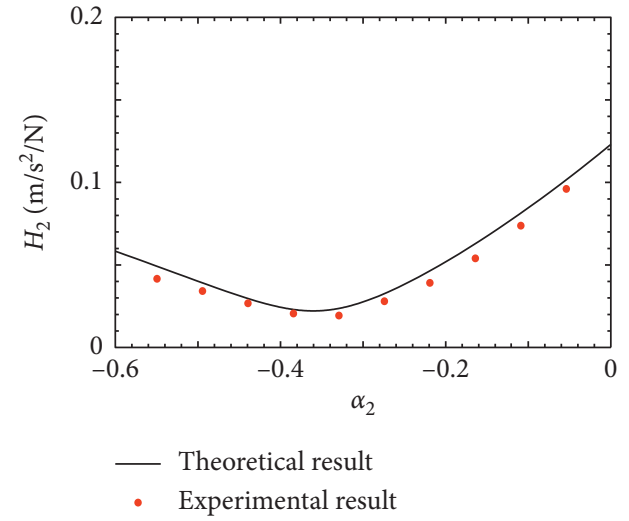

(b)

FigURE 6: $H_{i}(i=1,2)$ versus $\alpha_{2}$ for $\Omega=9.75 \mathrm{~Hz}, \alpha_{1}=-0.4$, and $\tau_{2}=50 \mathrm{~ms}$ : (a) absorber and (b) primary system.

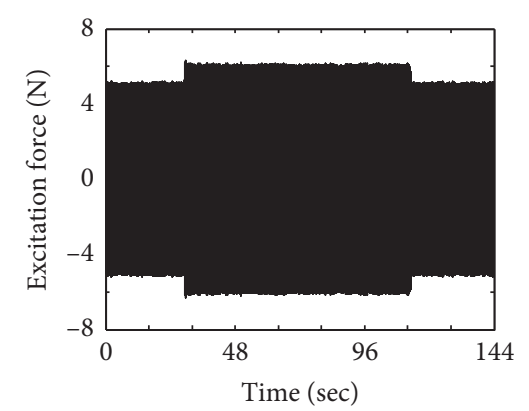

(a)

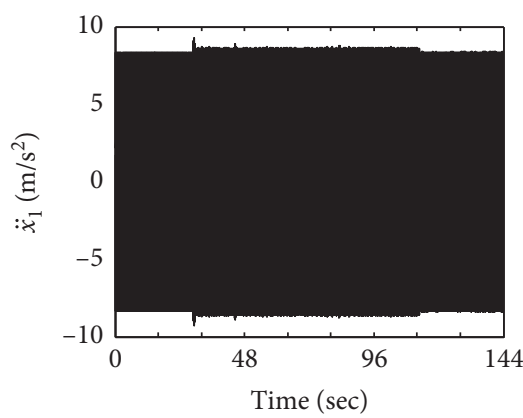

(d)

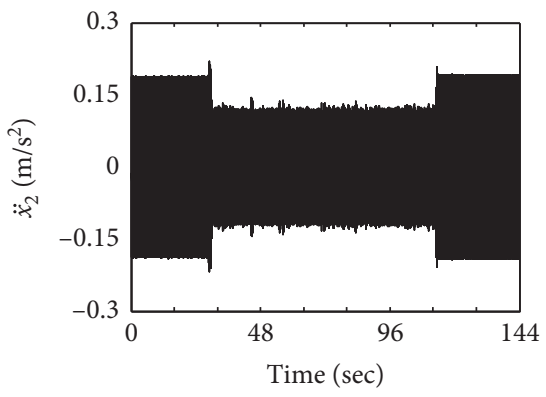

$(\mathrm{g})$

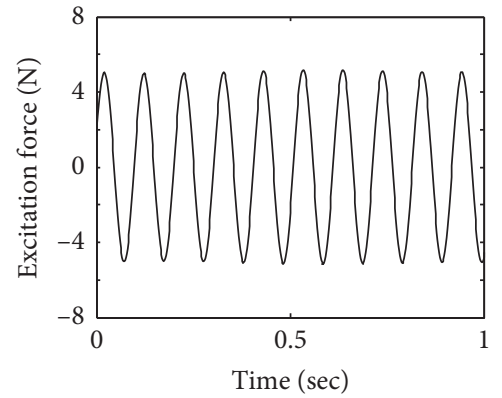

(b)

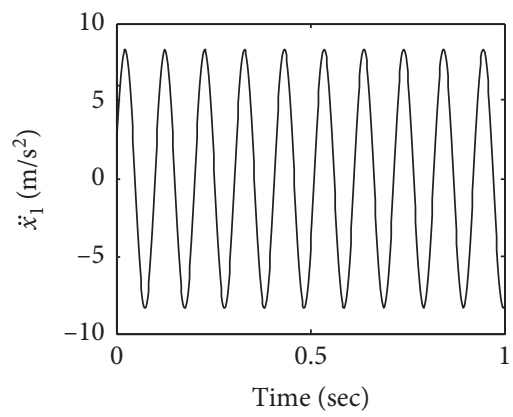

(e)

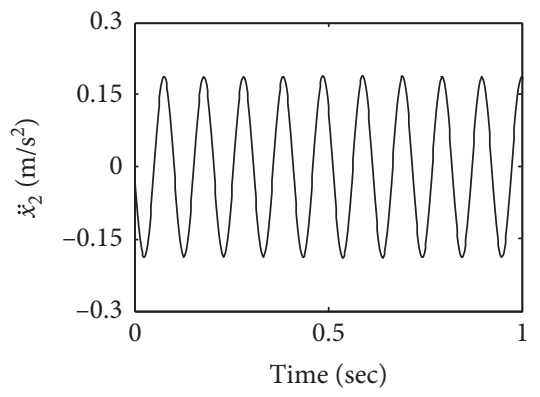

(h)

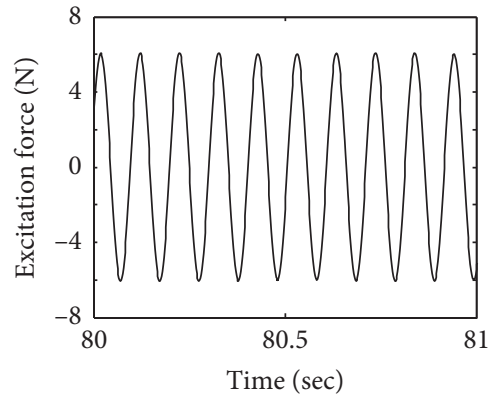

(c)

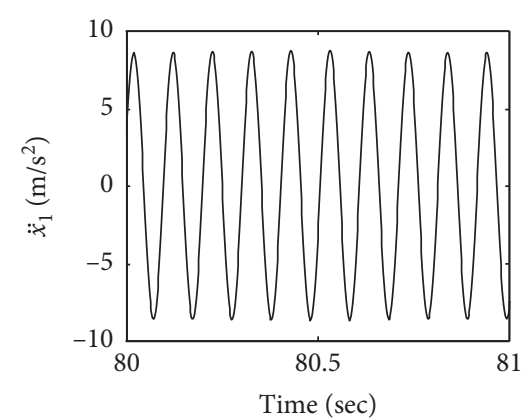

(f)

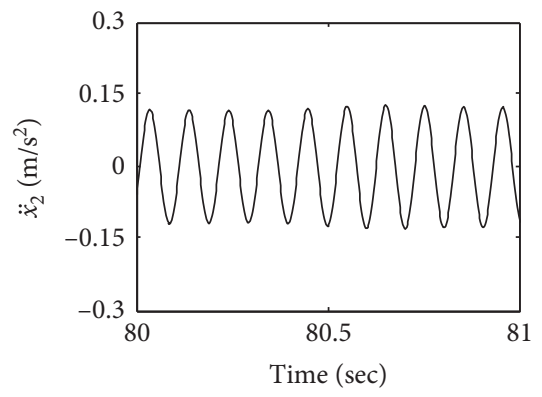

(i)

FIGURE 7: Measured time histories of excitation force and system accelerations when $\Omega=9.75 \mathrm{~Hz}$ (for $t<29 \mathrm{~s}$ and $t>113 \mathrm{~s}, \alpha_{1}=\alpha_{2}=0$; for $29 \mathrm{~s} \leq t \leq 113 \mathrm{~s}, \alpha_{1}=-0.4, \alpha_{2}=-0.33$, and $\tau_{2}=50 \mathrm{~ms}$ ): (a) excitation force; (d) absorber; (g) primary system; (b, c) zoom of (a); (e, f) zoom of (d); (h, i) zoom of (g). 
TABLE 2: Effect of $\alpha_{2}$ on the vibration suppression effect for $\Omega=9.75 \mathrm{~Hz}, \alpha_{1}=-0.4$, and $\tau_{2}=50 \mathrm{~ms}$.

\begin{tabular}{|c|c|c|c|c|c|}
\hline$\Omega(\mathrm{Hz})$ & $\tau_{2}(\mathrm{~ms})$ & $\alpha_{1}$ & $\alpha_{2}$ & $H_{2}\left(\mathrm{~m} / \mathrm{s}^{2} / \mathrm{N}\right)$ & $D(\%)$ \\
\hline 9.75 & - & 0 & 0 & 0.037 & 0 \\
\hline 9.75 & 50 & -0.4 & -0.33 & 0.020 & -45.95 \\
\hline 9.75 & 50 & -0.4 & -0.055 & 0.096 & 159.46 \\
\hline
\end{tabular}

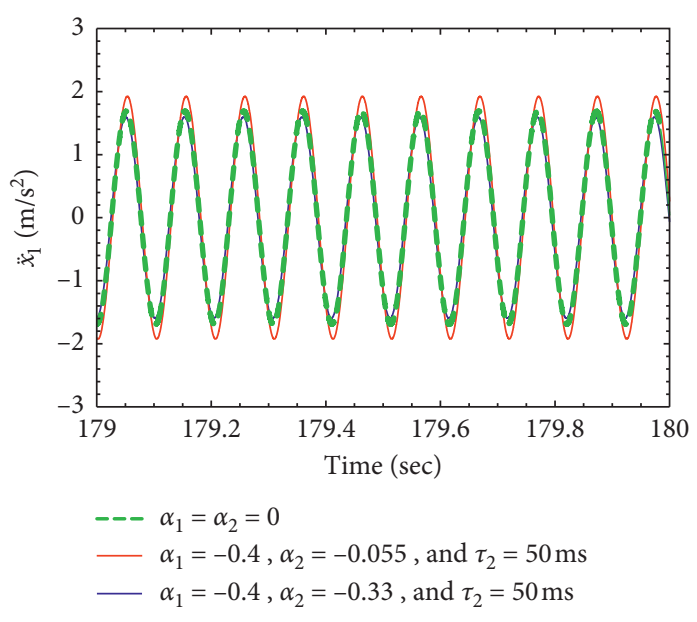

(a)

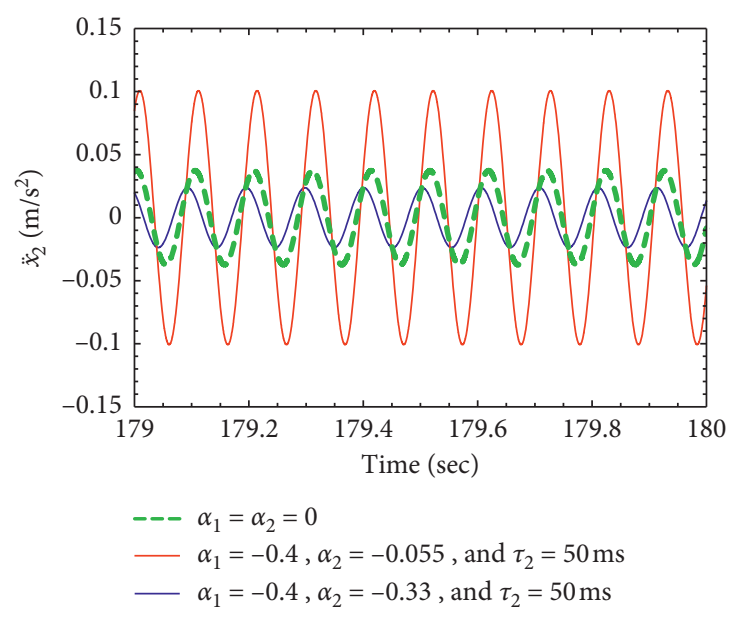

(b)

FIGURE 8: Numerical simulations of system responses for $\Omega=9.75 \mathrm{~Hz}$ and $f=1 \mathrm{~N}$ : (a) absorber and (b) primary system.

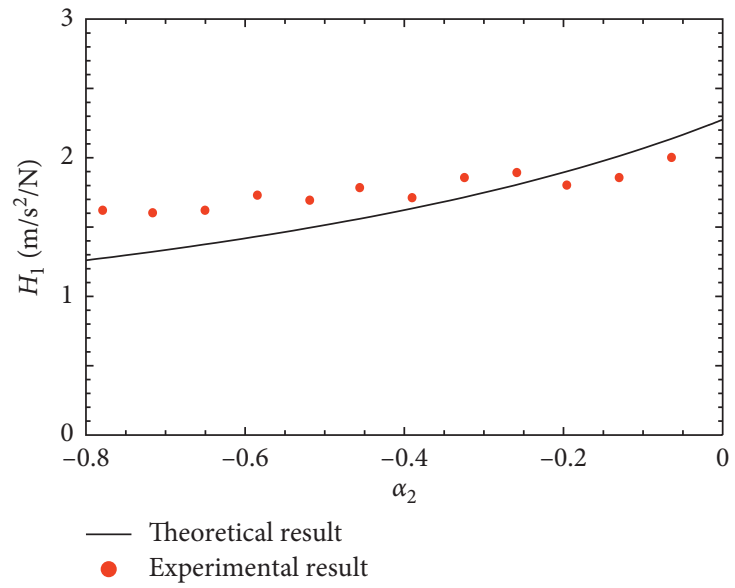

(a)

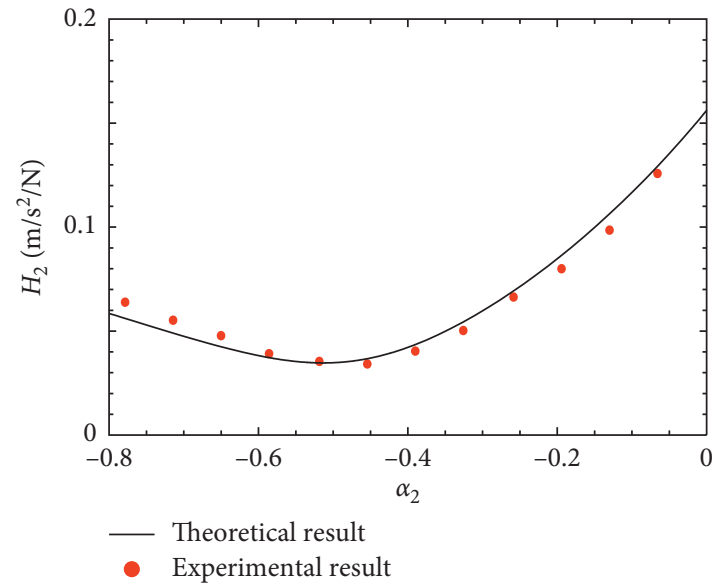

(b)

FiguRE 9: $H_{i}(i=1,2)$ versus $\alpha_{2}$ for $\Omega=10 \mathrm{~Hz}, \alpha_{1}=0.25$, and $\tau_{2}=30 \mathrm{~ms}$ : (a) absorber and (b) primary system.

absorber and the primary system, respectively, decrease $4.2 \%$ and $72.9 \%$.

Table 3 shows the values of $H_{2}$ under different values of feedback control parameters. It is attractive that the value of $H_{2}$ decreases $78.92 \%$ for $\alpha_{1}=0.25$ and $\alpha_{2}=-0.455$.

Figure 11 shows the numerical simulations of system acceleration responses for $\Omega=10 \mathrm{~Hz}$ and $f=1 \mathrm{~N}$, which are in agreement with the results shown in Figure 9 and Table 3.
3.2.2. Effect of $\tau_{2}$. The variations of $H_{i}(i=1,2)$ versus $\tau_{2}$ for $\Omega=10.25 \mathrm{~Hz}$ and $\alpha_{1}=\alpha_{2}=0.5$ are shown in Figure 12. It is clear that the values of $H_{i}(i=1,2)$ fluctuate as $\tau_{2}$ increases. The value of $H_{2}$ is maximal for $\tau_{2}=36 \mathrm{~ms}$ and minimal for $\tau_{2}=84 \mathrm{~ms}$. Figure 13 shows the measured time histories of excitation force and system acceleration for $\Omega=10.25 \mathrm{~Hz}$.

Table 4 shows the influence of $\tau_{2}$ on the vibration suppression effect for $\Omega=10.25 \mathrm{~Hz}$ and $\alpha_{1}=\alpha_{2}=0.5$. The 


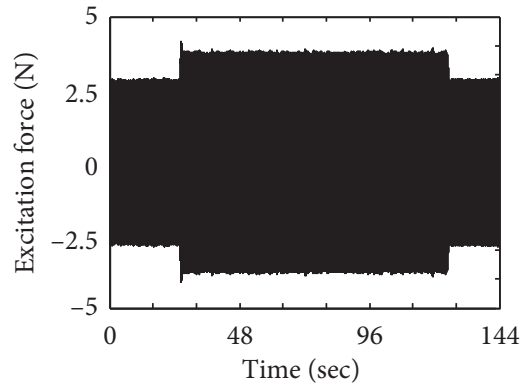

(a)

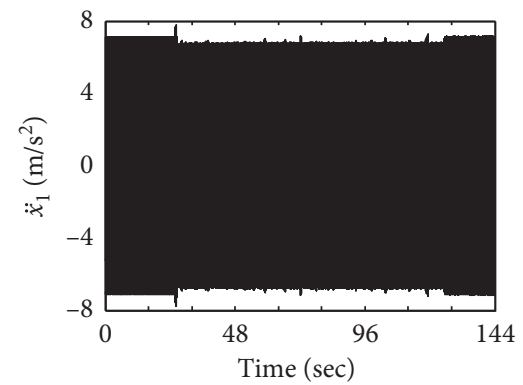

(d)

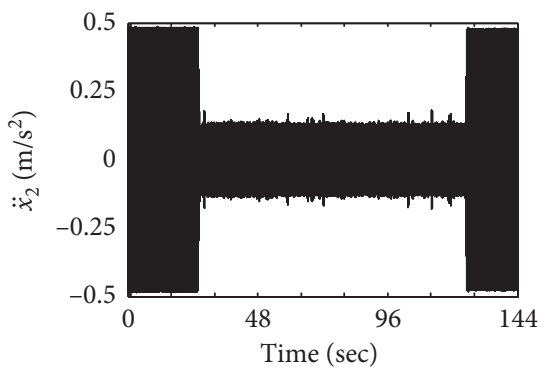

(g)

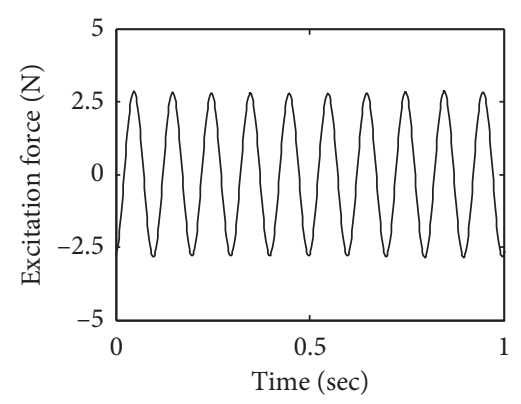

(b)

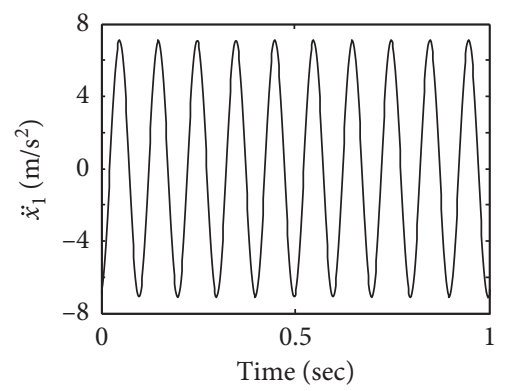

(e)

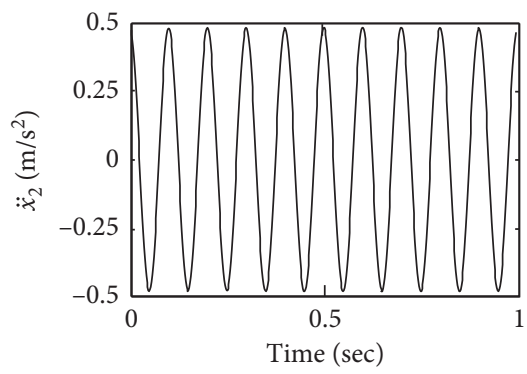

(h)

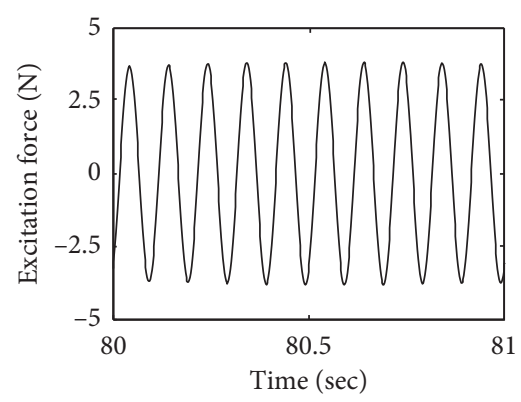

(c)

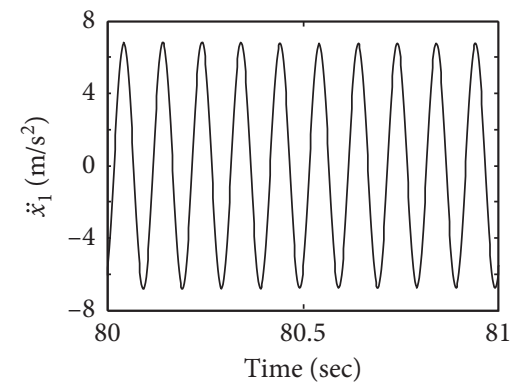

(f)

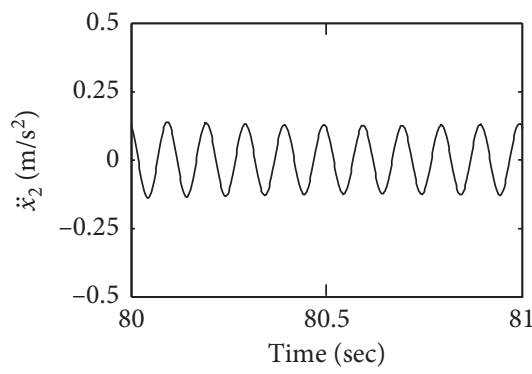

(i)

Figure 10: Measured time histories of excitation force and system acceleration when $\Omega=10 \mathrm{~Hz}$ (for $t<25 \mathrm{~s}$ and $t>125 \mathrm{~s}, \alpha_{1}=\alpha_{2}=0$; for $25 \mathrm{~s} \leq t \leq 125 \mathrm{~s}, \alpha_{1}=0.25, \alpha_{2}=-0.455$, and $\tau_{2}=30 \mathrm{~ms}$ ): (a) excitation force; (d) absorber; (g) primary system; (b, c) zoom of (a); (e, f) zoom of (d); (h, i) zoom of (g).

TABLE 3: Influence of $\alpha_{2}$ on the vibration suppression effect for $\Omega=10 \mathrm{~Hz}, \alpha_{1}=0.25$, and $\tau_{2}=30 \mathrm{~ms}$.

\begin{tabular}{lccccc}
\hline$\Omega(\mathrm{Hz})$ & $\tau_{2}(\mathrm{~ms})$ & $\alpha_{1}$ & $\alpha_{2}$ & $H_{2}\left(\mathrm{~m} / \mathrm{s}^{2} / \mathrm{N}\right)$ & 0.166 \\
\hline 10 & - & 0 & 0 & 0.035 & 0 \\
10 & 30 & 0.25 & -0.455 & -0.065 & 0.126 \\
10 & 30 & 0.25 & -24.10 \\
\hline
\end{tabular}

value of $\mathrm{H}_{2}$ decreases $69.33 \%$ for $\tau_{2}=84 \mathrm{~ms}$. However, it is undesirable that the time-delayed absorber greatly intensifies the vibration of the primary system for $\tau_{2}=36 \mathrm{~ms}$.
Figure 14 shows the numerical simulations of system responses for $\Omega=10.25 \mathrm{~Hz}$ and $f=1 \mathrm{~N}$, which coincide with the experimental and the theoretical results shown in Figure 12. 

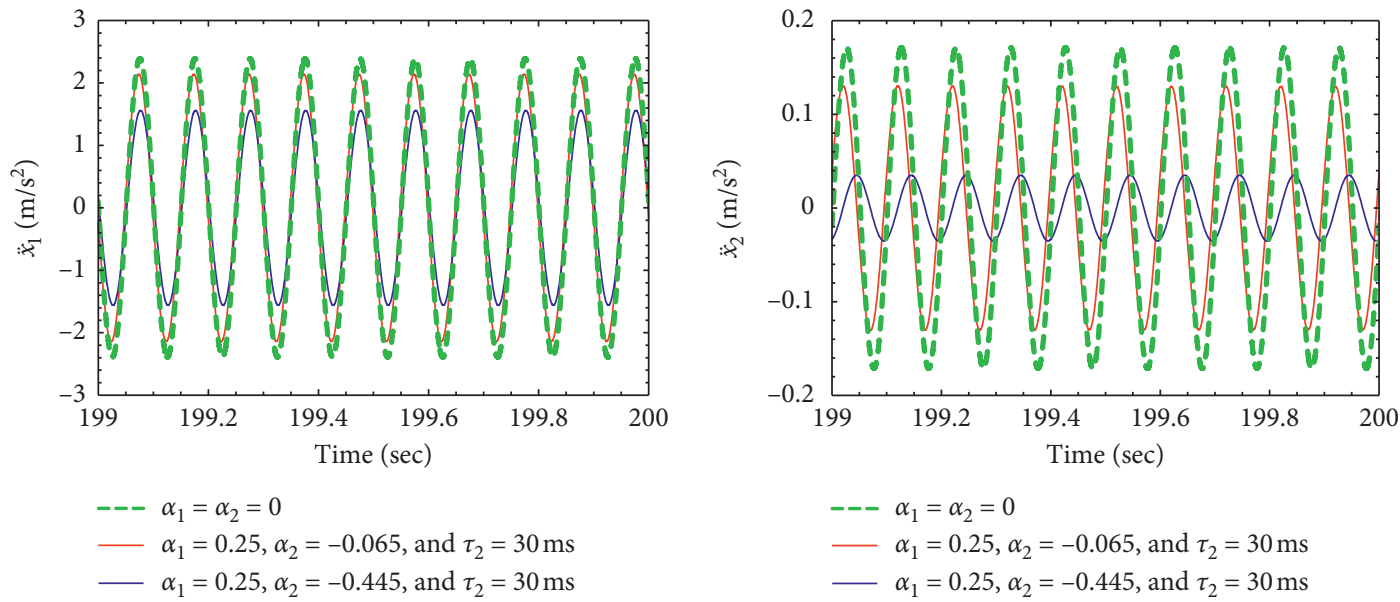

(b)

FIGURE 11: Numerical simulations of the time histories of system responses for $\Omega=10 \mathrm{~Hz}$ and $f=1 \mathrm{~N}$ : (a) absorber and (b) primary system.

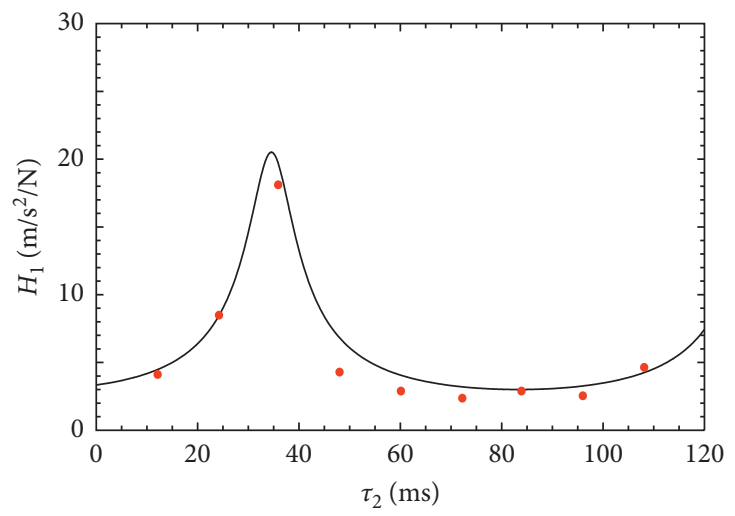

— Theoretical result

- Experimental result

(a)

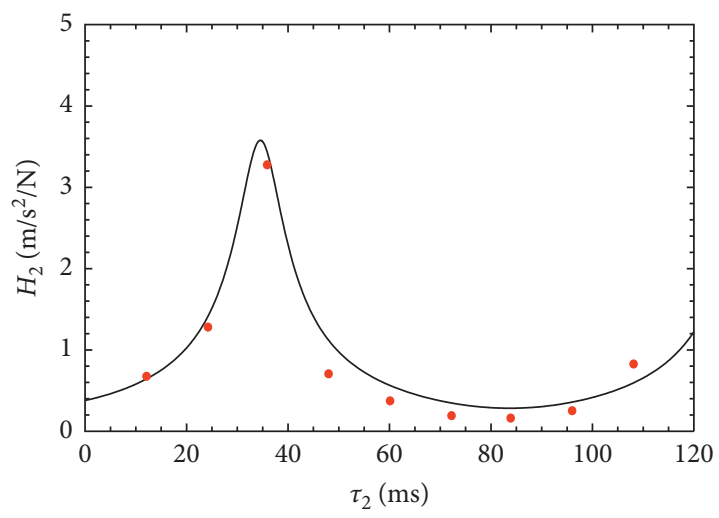

Theoretical result

- Experimental result

(b)

FIGURE 12: $H_{i}(i=1,2)$ versus $\tau_{2}$ for $\Omega=10.25 \mathrm{~Hz}$ and $\alpha_{1}=\alpha_{2}=0.5$ : (a) absorber and (b) primary system.

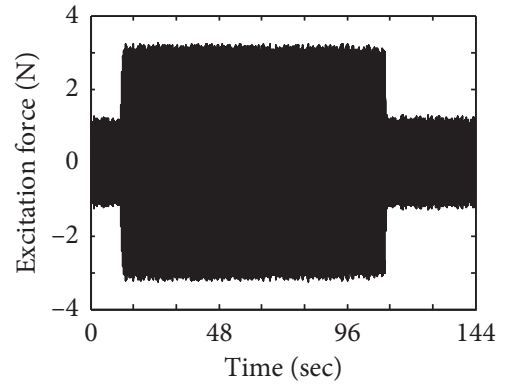

(a)

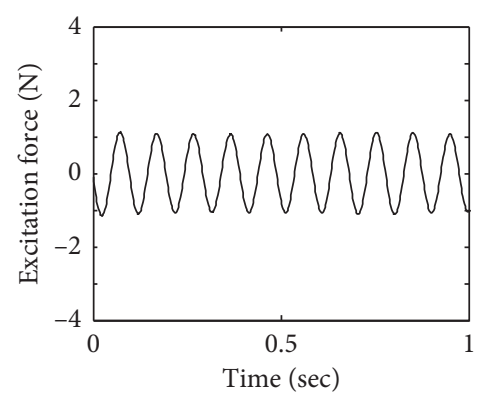

(b)

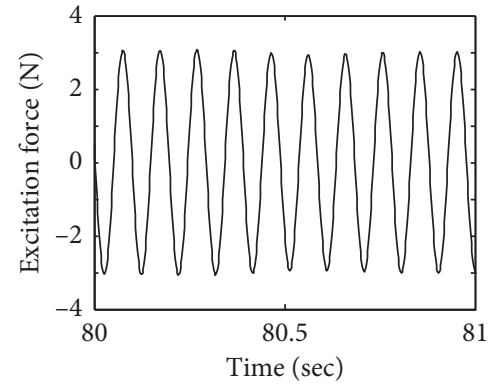

(c)

Figure 13: Continued. 


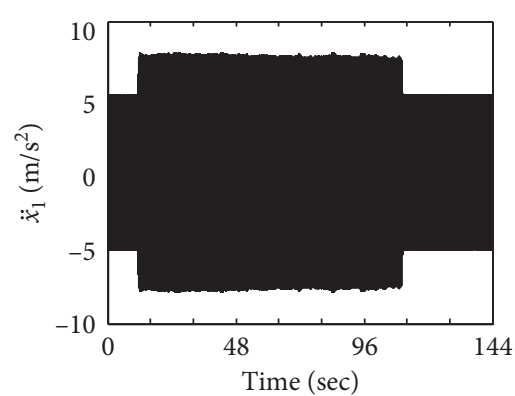

(d)

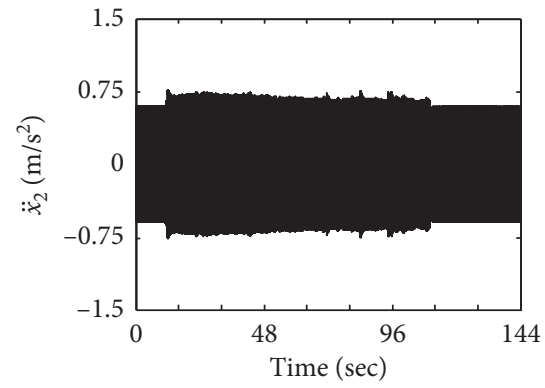

(g)

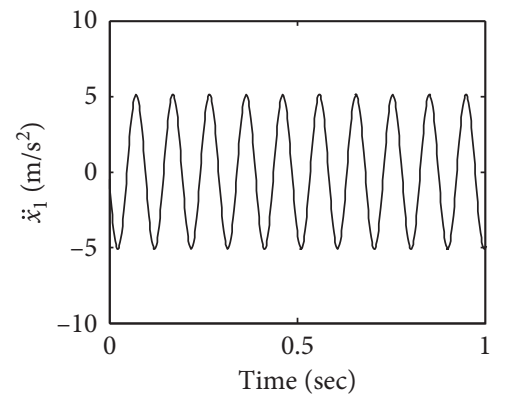

(e)

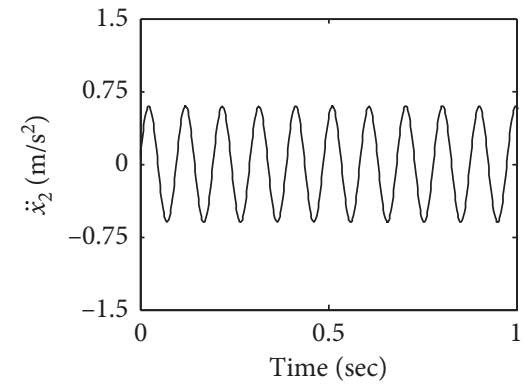

(h)

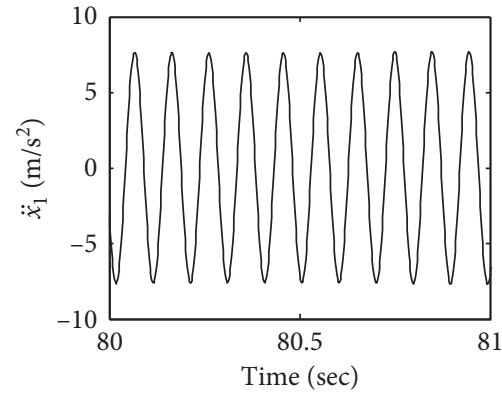

(f)

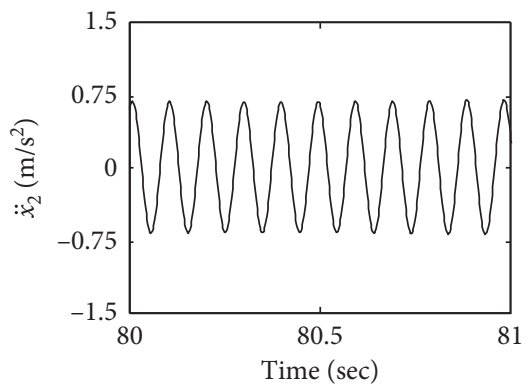

(i)

FIGURE 13: Time history of excitation force and system responses when $\Omega=10.25 \mathrm{~Hz}$ (for $t<11 \mathrm{~s}$ and $t>110 \mathrm{~s}, \alpha_{1}=\alpha_{2}=0$; for $11 \mathrm{~s} \leq t \leq 110 \mathrm{~s}$, $\alpha_{1}=\alpha_{2}=0.5$ and $\tau_{2}=96 \mathrm{~ms}$ ): (a) excitation force; (d) absorber; (g) primary system; (b, c) zoom of (a); (e, f) zoom of (d); (h, i) zoom of (g).

TABLE 4: Influence of $\tau_{2}$ on the vibration suppression effect for $\Omega=10.25 \mathrm{~Hz}$ and $\alpha_{1}=\alpha_{2}=0.5$.

\begin{tabular}{lccccc}
\hline$\Omega(\mathrm{Hz})$ & $\tau_{2}(\mathrm{~ms})$ & $\alpha_{1}$ & $\alpha_{2}$ & $H_{2}\left(\mathrm{~m} / \mathrm{s}^{2} / \mathrm{N}\right)$ & 0 \\
\hline 10.25 & - & 0 & 0 & 0.538 & \\
10.25 & 36 & 0.5 & 0.5 & 3.272 & 508.18 \\
10.25 & 84 & 0.5 & 0.5 & 0.165 & -69.33 \\
\hline
\end{tabular}

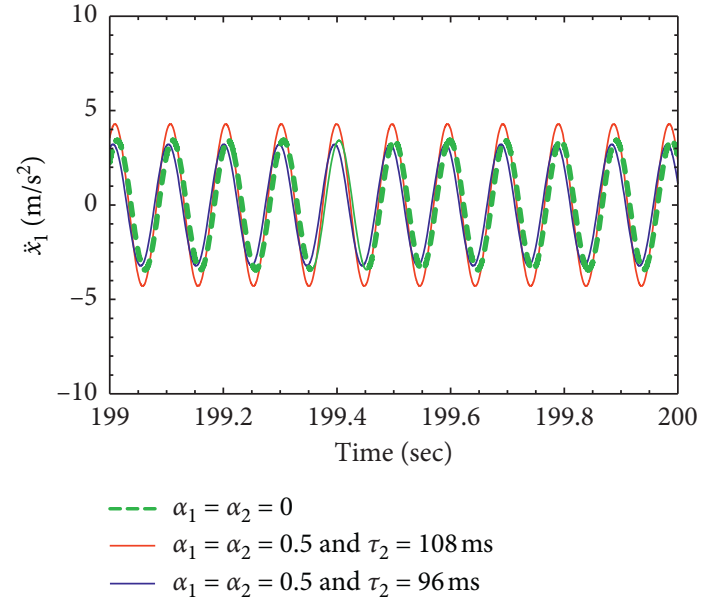

(a)

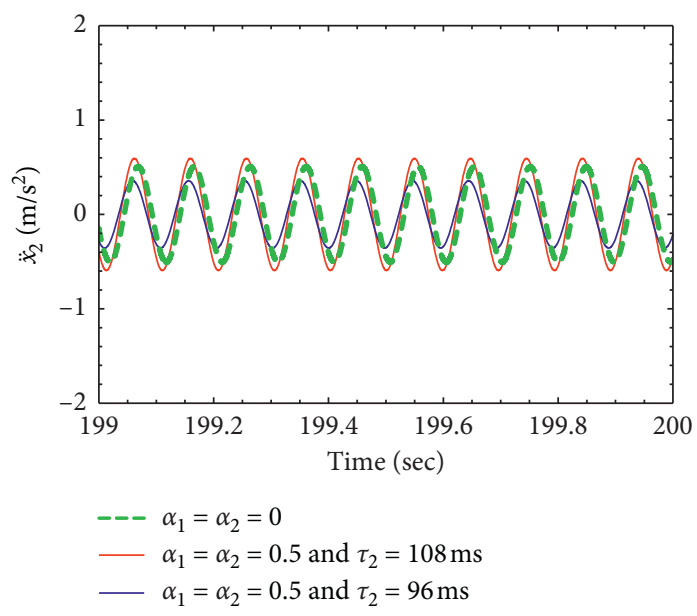

(b)

FIGURE 14: Numerical simulations of the time histories of system responses for $\Omega=10.25 \mathrm{~Hz}$ and $f=1 \mathrm{~N}$ : (a) absorber and (b) primary system. 


\section{Conclusions}

An active vibration suppression via a time-delayed absorber is presented. Case studies are provided to demonstrate the effects of feedback gain coefficient and intentional time delay on the vibration suppression performance of the timedelayed absorber. The following points are concluded:

(1) From the viewpoint of vibration suppression, the time-delayed absorber has advantages and disadvantages over the passive one, which depend on the values of the feedback gain coefficient and intentional time delay. The time-delayed absorber with proper choices of the two parameters decreases the values of acceleration transfer function of the primary system by $45.95 \%, 78.92 \%$, and $69.33 \%$ for $\Omega=9.75 \mathrm{~Hz}, 10 \mathrm{~Hz}$, and $10.25 \mathrm{~Hz}$, respectively. However, the time-delayed absorber fails when the value of the two parameters is improperly selected, which leads to the sharp vibration of the primary system.

(2) When the values of feedback gain coefficients are fixed, the value of intentional time delay determines the vibration suppression effect of the time-delayed absorber. It acts as a double-edged sword. Reasonable values of intentional time delay effectively improve the vibration suppression effect without changing the mass or stiffness of the absorber. However, unreasonable values of intentional time delay greatly intensify the vibration of the primary system. This situation should be avoided in practical engineering application.

\section{Appendix}

One has

$$
\begin{aligned}
d_{8}= & m_{1}^{2} m_{2}^{2}, \\
d_{6}= & 2 m_{1} m_{2} \cos \left(\omega \tau_{1}\right)\left[g_{1}\left(m_{1}+m_{2}\right)-g_{2} m_{2}\right]+2 c_{1} c_{2} m_{1}^{2}+c_{1}^{2}\left(m_{1}+m_{2}\right)^{2}+c_{2}^{2} m_{1}^{2}-2 m_{1} m_{2}\left[\left(k_{1}+k_{2}\right) m_{1}+k_{1} m_{2}\right] \\
d_{5}= & \left\{-2 c_{1} g_{2} m_{2}\left(m_{1}+m_{2}\right)+2 c_{1} g_{1}\left(m_{1}+m_{2}\right)^{2}+2 c_{2} g_{1} m_{1}^{2}\right\} \sin \left(\omega \tau_{1}\right) \\
d_{4}= & -2 c_{2}^{2} m_{1} k_{1}+m_{1}^{2}\left[g_{1}^{2}+\left(k_{1}+k_{2}\right)^{2}\right]+2 m_{1} m_{2}\left[g_{1}\left(g_{1}-g_{2}\right)+k_{1}\left(k_{1}+2 k_{2}\right)\right]+k_{1}^{2} m_{2}^{2}+m_{2}^{2} g_{1}\left(g_{1}-2 g_{2}\right)+c_{1}^{2}\left[c_{2}^{2}-2 k_{2}\left(m_{1}+m_{2}\right)\right] \\
& +2\left\{-g_{1} m_{1}^{2}\left(k_{1}+k_{2}\right)+2 g_{2} k_{2} m_{1} m_{2}+c_{2} m_{1}\left(c_{2} g_{1}-c_{1} g_{2}-c_{2} g_{2}\right)-2 g_{1} m_{1} m_{2}\left(k_{1}+k_{2}\right)+k_{1} m_{2}^{2}\left(g_{2}-g_{1}\right)\right\} \cos \left(\omega \tau_{1}\right) \\
& +g_{2} k_{1} m_{1} m_{2} \cos \left(\omega \tau_{1}\right) \\
d_{3}= & 2\left\{c_{1} c_{2}^{2}\left(g_{1}-g_{2}\right)-g_{2} m_{1}\left(c_{2} k_{1}-c_{1} k_{2}\right)-2 c_{1} g_{1} k_{2}\left(m_{1}+m_{2}\right)+2 c_{1} g_{2} k_{2} m_{2}\right\} \sin \left(\omega \tau_{1}\right) \\
d_{2}= & c_{2}^{2}\left(g_{1}^{2}-2 g_{1} g_{2}+k_{2}^{1}\right)+c_{1}^{2} k_{2}^{2}-2 g_{1}^{2} k_{2}\left(m_{1}+m_{2}\right)+2 g_{1} g_{2} k_{2}\left(m_{1}+2 m_{2}\right)-2 k_{1} k_{2}\left(k_{1} m_{1}+k_{2} m_{1}+k_{1} m_{2}\right) \\
& +2\left\{c_{1}^{2} k_{1}\left(g_{2}-g_{1}\right)+k_{2} m_{1}\left(-g_{2} k_{1}+g_{1} k_{2}-g_{2} k_{2}\right)+2 k_{1} k_{2}\left(g_{1} m_{1}+g_{1} m_{2}-g_{2} m_{2}\right)\right\} \cos \left(\omega \tau_{1}\right), \\
d_{1}= & 2 c_{1}\left(g_{1}-g_{2}\right) k_{2}^{2} \sin \left(\omega \tau_{1}\right), \\
d_{0}= & k_{2}^{1} k_{2}^{2}+2 k_{2}^{2} k_{1}\left(g_{2}-g_{1}\right) \cos \left(\omega \tau_{1}\right)+k_{2}^{2} g_{1}\left(g_{1}-2 g_{2}\right) .
\end{aligned}
$$

\section{Data Availability}

The data used to support the findings of this study are available from the corresponding author upon request.

\section{Disclosure}

This work was based on the manuscript presented in the 9th European Nonlinear Dynamics Conference.

\section{Conflicts of Interest}

The author declares that there are no conflicts of interest regarding the publication of this paper.

\section{Acknowledgments}

This work was supported by the National Natural Science Foundation of China under Grant no. 11602135.

\section{References}

[1] F. E. Udwadia and P. Phohomsiri, "Active control of structures using time delayed positive feedback proportional control designs," Structural Control and Health Monitoring, vol. 13, no. 1, pp. 536-552, 2006.

[2] B. Xu, W. Zhang, and J. Ma, "Stability and Hopf bifurcation of a two-dimensional supersonic airfoil with a time-delayed feedback control surface," Nonlinear Dynamics, vol. 77, no. 3, pp. 1-19, 2014

[3] H. Zhang, H. Chen, C. Jiang, and K. Wang, "Effect of explicit dynamics of free virus and intracellular delay," Chaos, Solitons \& Fractals, vol. 104, pp. 827-834, 2017.

[4] R. A. Delgado, K. Lau, R. H. Middleton, and T. Wigren, "Networked delay control for 5G wireless machine-type communications using multiconnectivity," IEEE Transactions on Control Systems Technology, vol. 27, no. 4, pp. 1510-1525, 2019.

[5] Y. Yan and J. Xu, "Suppression of regenerative chatter in a plunge-grinding process by spindle speed," Journal of 
Manufacturing Science and Engineering, vol. 135, no. 4, 9 pages, Article ID 041019, 2013.

[6] L. L. Chung, A. M. Reinhorn, and T. T. Soong, "Experiments on active control of seismic structures," Journal of Engineering Mechanics, vol. 114, no. 2, pp. 241-256, 1988.

[7] J. E. Normey-Rico, P. Garcia, and A. Gonzalez, "Robust stability analysis of filtered Smith predictor for time-varying delay processes," Journal of Process Control, vol. 22, no. 10, pp. 1975-1984, 2012.

[8] A. K. Agrawal and J. N. Yang, "Compensation of time-delay for control of civil engineering structures," Earthquake Engineering \& Structural Dynamics, vol. 29, no. 1, pp. 37-62, 2000.

[9] M. Xiao and J. Cao, "Bifurcation analysis and chaos control for lü system with delayed feedback," International Journal of Bifurcation and Chaos, vol. 17, no. 12, pp. 4309-4322, 2007.

[10] Y. Chen, S. Fei, and Y. Li, "Stabilization of neutral time-delay systems with actuator saturation via auxiliary time-delay feedback," Automatica, vol. 52, pp. 242-247, 2015.

[11] Y. A. Amer, A. T. EL-Sayed, and A. A. Kotb, "Nonlinear vibration and of the Duffing oscillator to parametric excitation with time delay feedback," Nonlinear Dynamics, vol. 85, no. 4, pp. 2497-2505, 2016.

[12] J. Xu, Y. Chen, and K. W. Chung, "An improved time-delay saturation controller for suppression of nonlinear beam vibration," Nonlinear Dynamics, vol. 82, no. 4, pp. 1691-1707, 2015.

[13] N. Olgac and B. T. Holm-Hansen, "A novel active vibration absorption technique: delayed resonator," Journal of Sound and Vibration, vol. 176, no. 1, pp. 93-104, 1994.

[14] D. Filipovic and N. Olgac, "Delayed resonator with speed feedback-design and performance analysis," Mechatronics, vol. 12, no. 3, pp. 393-413, 2002.

[15] V. Kucera, D. Pilbauer, T. Vyhlidal et al., "Extended delayed resonators - design and experimental verification," Mechatronics, vol. 41, pp. 29-44, 2017.

[16] D. Pilbauer, T. Vyhlidal, and N. Olgac, "Delayed resonator with distributed delay in acceleration feedback-design and experimental verification," IEEE/ASME Transactions on Mechatronics, vol. 21, no. 4, pp. 2120-2131, 2016.

[17] Y. Y. Zhao and J. Xu, "Effects of delayed feedback control on nonlinear vibration absorber system," Journal of Sound and Vibration, vol. 308, no. 1-2, pp. 212-230, 2007.

[18] S. Mohanty and S. K. Dwivedy, "Nonlinear dynamics of piezoelectric-based active nonlinear vibration absorber using time delay acceleration feedback," Nonlinear Dynamics, vol. 98, no. 2, pp. 1465-1490, 2019.

[19] X. T. Sun and Y. S. Song, "Dynamical performances of a vibration absorber for continuous structure considering timedelay coupling," Shock and Vibraiton, vol. 2016, Article ID 5039796, 15 pages, 2016.

[20] F. Wang and J. Xu, "Parameter design for a vibration absorber with time-delayed feedback control," Acta Mechanica Sinica, vol. 35, no. 3, pp. 624-640, 2019.

[21] X. Zhang, J. Xu, and J. Ji, "Modelling and tuning for a timedelayed vibration absorber with friction," Journal of Sound and Vibration, vol. 424, pp. 137-157, 2018.

[22] Y. Sun and J. Xu, "Experiments and analysis for a controlled mechanical absorber considering delay effect," Journal of Sound and Vibration, vol. 339, pp. 25-37, 2015.

[23] Y. X. Sun, "Design and experimental studies of an active vibration absorber with adjustable time delay," in Proceedings of the 2017 International Conference on Advanced Mechatronic Systems, pp. 386-391, Xiamen, China, December 2017. 